\title{
UPDATE
}

\section{Update of trichomoniasis}

\section{J R Schwebke}

Trichomoniasis remains an extremely common infection despite the fact that rates of other treatable sexually transmitted diseases are declining. Newer diagnostic techniques such as polymerase chain reaction (PCR) are documenting higher rates of infection in heterosexual men than have been previously found with culture. Although data on the association of vaginal trichomoniasis with preterm birth are controversial, the association of trichomoniasis with HIV acquisition seems clear. Despite being a readily diagnosed and treated STD, trichomoniasis is not a reportable infection and control of the infection has received relatively little emphasis from public health STD control programmes. More recently, however, appreciation of high rates of disease and of associations of trichomoniasis in women with adverse outcomes of pregnancy and increased risk for HIV infection suggest a need for increased control efforts.

Correspondence to:

J R Schwebke, University of Alabama at Birmingham, 703 19th Street, South Zeigler Research Building No 239, Birmingham, AL 35294-0007, USA; Schwebke@uab.edu

Accepted for publication 4 July 2002
$T$ he World Health Organization (WHO) has estimated that this infection accounts for almost half of all curable sexually transmitted infections worldwide. ${ }^{1}$ The annual incidence of Trichomonas vaginalis infections in the United States has been estimated at five million cases. Epidemiologically, $T$ vaginalis infections are commonly associated with other STDs and may be a particularly sensitive marker of high risk sexual behaviour. Trichomoniasis is frequently seen concomitantly with other STDs, particularly gonorrhoea. ${ }^{2}$ The majority of women with trichomoniasis also have bacterial vaginosis $(\mathrm{BV}) .^{3-5}$ Unlike other STDs which have higher prevalence among adolescents and young adults, rates of trichomoniasis are more evenly distributed among sexually active women of all age groups. ${ }^{2}$

Trichomonas vaginalis, a flagellated parasite, is the causative agent of this infection. Although there are two additional species of Trichomonas that infect humans ( $T$ tenax and $T$ hominis), $T$ vaginalis is the only one that infects the urogenital tract. Symptoms of trichomoniasis in women include vaginal discharge, irritation, and pruritus; however, about half of all women infected with $T$ vaginalis are asymptomatic. ${ }^{6}$ Signs of infection in women include vaginal discharge, odour, and oedema or erythema. In males, the prevalence and spectrum of disease is far less well characterised; the infection appears usually to be asymptomatic. However, it has been suggested as an increasingly important cause of non-gonococcal urethritis (NGU). ${ }^{7}$ A recent study, using polymer- ase chain reaction (PCR) for diagnosis, found $T$ vaginalis in $17 \%$ of men attending an STD clinic in Birmingham, Alabama (Schwebke et al, unpublished data).

Diagnosis of trichomoniasis in the female is usually accomplished via direct microscopic examination of the vaginal fluid; however, even with skilled diagnosticians the sensitivity of this test is only $60 \%$ overall and may be less in asymptomatic women. ${ }^{8}$ Culture media are commercially available and are currently the gold standard for diagnosis. ${ }^{9}$ Polymerase chain reaction (PCR) techniques are under development but have thus far shown variable results, especially in women. ${ }^{10-14}$ Diagnosis in general is much more difficult for males with the best culture results yielded by combining urethral swabs and urine sediment. ${ }^{7}$ A recent comparison study of culture versus PCR in men showed significantly greater sensitivity for the latter (Schwebke et al, unpublished data).

In most cases, trichomoniasis is easily treated with a single dose of metronidazole and because it is an STD sexual partners should be routinely treated. ${ }^{15}$ However, because of the mainly asymptomatic nature of the infection in males it is doubtful that partner referral is frequently successful. Resistant cases appear to occur sporadically. The prevalence of resistant strains is unknown but it may be increasing in prevalence. ${ }^{16}$ The resistance is relative and can usually be overcome with increased doses of metronidazole. ${ }^{17}$ Tinidazole appears to be an attractive alternative to metronidazole. It has a longer half life than metronidazole and has been effective in some cases of trichomoniasis that were resistant to metronidazole. ${ }^{18}$

Long considered a "minor" STD with few associated complications, infection with $T$ vaginalis has recently been implicated as a cause of preterm delivery in several studies. In a large multicentre study, after adjusting for demographic, behavioural, and microbiological variables, trichomonas was significantly associated with low birth weight, premature rupture of membranes, and preterm delivery $(\mathrm{RR}=1.4) .{ }^{19}$ Similarly, Minkoff et al also documented a significant correlation between trichomoniasis and premature rupture of membranes. In the Minkoff study, the incidence of this complication at term was $27.5 \%$ in women with trichomonas versus $12.8 \%$ in those without $(\mathrm{p}<0.03) .{ }^{20}$ In another study of pregnant adolescents, $T$ vaginalis was independently associated with prematurity and low birth weight. ${ }^{21} \mathrm{~A}$ more recent randomised treatment study has, however, lent controversy to this area. Klebanoff et al reported the results of a study designed to prevent preterm labour among women with trichomoniasis. Women with asymptomatic trichomonal infection were randomised to placebo versus 
treatment with metronidazole. The latter was given as a $2 \mathrm{~g}$ dose at four separate intervals during the pregnancy - that is, at four times the normal treatment dose for this infection. Unexpectedly, women in the treatment group were found to have higher rates of preterm birth than those who received placebo. ${ }^{22}$ The results of this study may be difficult to extrapolate to the clinical care of the pregnant woman with trichomoniasis as only women with asymptomatic infections were included in the study and much higher doses of metronidazole were used than is the standard of care. It is possible that the latter in some manner had an impact on preterm birth rates. It is also possible that women with symptomatic trichomoniasis may have a different response to treatment than women with asymptomatic infection as a result of organism burden or host factors. In light of the cross sectional data, further studies may be warranted to resolve the conflicting findings.

Acquisition of the human immunodeficiency virus (HIV) has been associated with trichomoniasis in several African studies, possibly as a result of local inflammation often caused by the parasite. Leroy et al found a significant difference between the prevalence of trichomoniasis among a cohort of HIV infected and non-infected pregnant women in Rwanda $(20.2 \% v 10.9 \%, \mathrm{p}=0.0007) .{ }^{23}$ In a prospective study by Laga $e t$ al, incident trichomoniasis was significantly associated with HIV seroconversion $(\mathrm{OR}=1.9)$ among a cohort of women in Zaire in multivariate analysis. ${ }^{24}$ Buve et al reported significantly higher rates of vaginal trichomoniasis among women residing in high HIV prevalence cities than in low HIV prevalence cities and suggested that trichomoniasis may be an important factor in determining rates of HIV. ${ }^{25}$ The associations between HIV and trichomoniasis, as well as other STDs, may relate to (1) increased shedding of HIV as a result of the local inflammation produced by the STD, (2) increased susceptibility to HIV as a result of the macroscopic or microscopic breaks in mucosal barriers caused by the STD, (3) STDs may be more prevalent among HIV infected individuals as a result of common risk factors for both infections, or (4) the immunosuppression associated with HIV infection may lead to increased susceptibility to STDs. Given the higher prevalence and incidence of trichomoniasis than most other treatable STDs in most studies to date, the attributable fraction of HIV acquisitions due to trichomoniasis may eclipse the relative contribution of other STDs. ${ }^{26}$

\section{SUMMARY}

Despite the continued high numbers of cases and the potential complications of infection, trichomoniasis remains ignored as a public health issue. The only settings in which this infection is routinely screened for is in public health STD clinics and the screening test used is the vaginal wet preparation which has limited sensitivity. Successful control of STDs is greatly aided by the use of a sensitive screening test, treatment of infected partners, and the availability of effective single dose medication. To date, only the latter is widely available for trichomoniasis.

\section{REFERENCES}

1 Cates W, and the American Social Health Association Panel. Estimates of the incidence and prevalence of sexually transmitted diseases in the United States. Sex Transm Dis 1999;26:52-7.

2 Lossick JG. Epidemiology of urogenital trichomoniasis. In: Trichomonads parasitic in humans, 1989:311-23.

3 James JA, Thomason JL, Gelbart SM, et al. Is trichomoniasis often associated with bacterial vaginosis in pregnant adolescents? Am J Obstet Gynecol 1992;166:859-62.

4 Thomason JL, Gelbart SM, Scaglione JJ. Bacterial vaginosis: current review with indication for asymptomatic women. Am J Obstet Gynecol 1991:165:1210-17.

5 Wølner-Hanssen P, Krieger JN, Stevens CE, et al. Clinical manifestations of vaginal trichomoniasis. JAMA 1989;261:571-6.

6 Fouts AC, Kraus SJ. Trichomonas vaginalis: re-evaluation of its clinical presentation and laboratory diagnosis. J Infect Dis 1980;141:137-43.

7 Krieger JN, Verdon M, Siegel N, et al. Natural history of urogenital trichomoniasis in men. Urology 1993;149:1455-8.

8 Krieger JN, Tam MR, Stevens CE, et al. Diagnosis of trichomoniasis. JAMA 1988;259:1223-7.

9 Draper D, Parker R, Patterson E, et al. Detection of Trichomonas vaginalis in pregnant women with the InPouch TV system. J Clin Microbiol 1993;31:1016-18.

10 Lawing L, Hedges S, Schwebke J. Detection of trichomonosis in vaginal and urine specimens from women by culture and PCR. J Clin Microbiol 2000;38:3585-8

11 Madico G, Quinn TC, Rompalo A, et al. Diagnosis of Trichomonas vaginalis infection by PCR using vaginal swab samples. J Clin Microbiol 1998;36:3205-10

12 Heine RP, Wiensfeld HC, Sweet RL, et al. Polymerase chain reaction analysis of distal vaginal specimens: a less invasive strategy for detection of Trichomonas vaginalis. Clin Infect Dis 1997:24:985-7.

13 Riley DE, Roberts MC, Takayama T, et al. Development of a polymerase chain reaction based diagnosis of Trichomonas vaginalis. J Clin Microbiol 1992;30:465-72.

14 Hobbs M, Kazembe P, Reed A, et al. Trichomonas vaginalis as a cause of urethritis in Malawian men. Sex Transm Dis 1999;26:381-7.

15 CDC. 1998 Guidelines for treatment of sexually transmitted diseases. MMWR 1998:47(RR-1).

16 Sobel J, Nagappan V, Nyirjesy P. Metronidazole-resistant vaginal trichomoniasis - an emerging problem. N Engl J Med 1999;341:292-3.

17 Lossick JG, Muller M, Gorrell TE. In vitro drug susceptibility and doses of metronidazole required for cure in cases of refractory vaginal trichomoniasis. J Infect Dis 1986;153:948-55.

18 Lossick JG, Kent HL. Trichomoniasis: trends in daignosis and management. Am J Obstet Gynecol 1991;165:1217-22.

19 Cotch MF, Pastorek JG, Nugent RP, et al. Trichomonas vaginalis associated with low birth weight and preterm delivery. Sex Transm Dis 1997:24:341-61

20 Minkoff H, Grunebaum AN, Schwarz RH, et al. Risk factors for prematurity and premature rupture of membranes: a prospective study of the vaginal flora in pregnancy. Am J Obstet Gynecol 1984;150:96572.

21 Hardy P, Hardy J, Nell E, Graham D. Prevalence of six sexually transmitted disease agents among pregnant inner-city adolescents and pregnancy outcome. Lancet 1984;2:333-7.

22 Klebanoff $M$, Carey J, Hauth JC, et al. Failure of metronidazole to prevent preterm delivery among pregnant women with asymptomatic Trichomonas vaginalis infection. N Engl J Med $2001 ; 345: 487-93$.

23 Leroy V, De Clercq A, Ladner J, et al. Should screening of genital infections be part of antenatal care in areas of high HIV prevalence? A prospective cohort study from Kigali, Rwanda, 1992-1993. Genitourin Med 1995; 71:207-11.

24 Laga M, Manoka A, Kivuvu M, et al. Non-ulcerative sexually transmitted diseases as risk factors for HIV-1 transmission in women: results from a cohort study. AIDS 1993;7:95-102.

25 Buve A, Weiss $H$, Laga $M$, et al. The epidemiology of trichomoniasis in women in four African cities. AIDS 2001;(Suppl 4):S89-96.

26 Sorvillo F, Kerndt P. Trichomonas vaginalis and amplification of HIV-1 transmission. Lancet 1998;351:213-14. 